\title{
Phase I trial of donor-derived modified immune cell infusion in kidney transplantation
}

Christian Morath, ${ }^{1,2}$ Anita Schmitt, ${ }^{3,2}$ Christian Kleist, ${ }^{4,5}$ Volker Daniel, ${ }^{4}$ Gerhard Opelz, ${ }^{4}$ Caner Süsal, ${ }^{4}$ Eman Ibrahim, ${ }^{4}$ Florian Kälble, ${ }^{1}$ Claudius Speer, ${ }^{1}$ Christian Nusshag, ${ }^{1}$ Luiza Pego da Silva, ${ }^{1,2}$ Claudia Sommerer, ${ }^{1}$ Lei Wang, ${ }^{3,2}$ Ming Ni, ${ }^{3}$ Angela Hückelhoven-Krauss, ${ }^{3}$ David Czock, ${ }^{6}$ Uta Merle, ${ }^{7}$ Arianeb Mehrabi, ${ }^{8}$ Anja Sander, ${ }^{9}$ Matthes Hackbusch, ${ }^{9}$ Christoph Eckert, ${ }^{10}$ Rüdiger Waldherr, ${ }^{10}$ Paul Schnitzler, ${ }^{11}$ Carsten Müller-Tidow, ${ }^{3}$ Jörg D. Hoheisel, ${ }^{12}$ Shakhawan A. Mustafa, ${ }^{12,13}$ Mohamed S.S. Alhamdani, ${ }^{12}$ Andrea S. Bauer, ${ }^{12}$ Jochen Reiser, ${ }^{14}$ Martin Zeier, ${ }^{1}$ Michael Schmitt, ${ }^{3}$ Matthias Schaier, ${ }^{1,2}$ and Peter Terness ${ }^{4}$

'Department of Nephrology, Heidelberg University Hospital, Heidelberg, Cermany. ${ }^{2}$ TolerogenixX GmbH, Heidelberg, Cermany. ${ }^{3}$ Department of Hematology, Oncology and Rheumatology, ${ }^{4}$ Transplantation Immunology, Institute of Immunology, ${ }^{5}$ Department of Nuclear Medicine, ${ }^{6}$ Department of Clinical Pharmacology and Pharmacoepidemiology, ${ }^{7}$ Department of Gastroenterology, ${ }^{8}$ Department of Ceneral, Visceral and Transplantation Surgery, Institute of Medical Biometry and Informatics, ${ }^{10}$ Institute of Pathology, and "Virology, Center for Infectious Diseases, Heidelberg University Hospital, Heidelberg, Germany. ${ }^{12}$ Division of Functional Genome Analysis, DKFZ, Heidelberg, Germany. ${ }^{13}$ Kurdistan Institution for Strategic Studies and Scientific Research, Kurdistan Region, Iraq. ${ }^{14}$ Department of Medicine, Rush Medical College, Rush University, Chicago, Illinois, USA.

BACKGROUND. Preclinical experiments have shown that donor blood cells, modified in vitro by an alkylating agent (modified immune cells [MICs]), induced long-term specific immunosuppression against the allogeneic donor.

METHODS. In this phase I trial, patients received either $1.5 \times 10^{6} \mathrm{MICs}$ per $\mathrm{kg}$ BW on day $-2\left(n=3\right.$, group A), or $1.5 \times 10^{8}$ MICs per kg BW on day -2 ( $n=3$, group B) or day $-7(n=4$, group C) before living donor kidney transplantation in addition to posttransplantation immunosuppression. The primary outcome measure was the frequency of adverse events (AEs) until day 30 (study phase) with follow-up out to day 360.

RESULTS. MIC infusions were extremely well tolerated. During the study phase, 10 treated patients experienced a total of 69 AEs that were unlikely to be related or not related to MIC infusion. No donor-specific human leukocyte antigen Abs or rejection episodes were noted, even though the patients received up to $1.3 \times 10^{10}$ donor mononuclear cells before transplantation. Group $C$ patients with low immunosuppression during follow-up showed no in vitro reactivity against stimulatory donor blood cells on day 360 , whereas reactivity against third-party cells was still preserved. Frequencies of CD19+CD24 ${ }^{\text {hi CD } 38^{\text {hi }}}$ transitional B lymphocytes (Bregs) increased from a median of $6 \%$ before MIC infusion to $20 \%$ on day 180 , which was 19- and 68-fold higher, respectively, than in 2 independent cohorts of transplanted controls. The majority of Bregs produced the immunosuppressive cytokine IL-10. MIC-treated patients showed the Immune Tolerance Network operational tolerance signature.

CONCLUSION. MIC administration was safe and could be a future tool for the targeted induction of tolerogenic Bregs.

TRIAL REGISTRATION. EudraCT number: 2014-002086-30; ClinicalTrials.gov identifier: NCT02560220.

FUNDING. Federal Ministry for Economic Affairs and Technology, Berlin, Germany, and TolerogenixX GmbH, Heidelberg, Germany.

Related Commentary: p. 2189

Authorship note: CM and A. Schmitt are co-first authors. M. Schaier and PT are co-senior authors.

Conflict of interest: CM, A. Schmitt, CK, GO, MZ, M. Schmitt, M. Schaier, and PT, together with the University of Heidelberg, are cofounders of TolerogenixX GmbH, a biotechnology company that holds licenses for modified immune cell (MIC) treatment. CK, GO, and PT hold a patent for MIC treatment ("Immunosuppressive blood cells and methods of producing the same." Patent no. WO 2010/000730, EP 2318020). CM, A. Schmitt, CK, VD, GO, C. Süsal, MZ, M. Schmitt, M. Schaier, and PT filed a patent application for MIC treatment ("MIC therapy for specific immunosuppression in transplantation." Patent no. PCT/EP2019/062857). JR is cofounder and shareholder of Trisaq, a biopharmaceutical company that develops novel therapies for kidney diseases.

Role of funding source: The funding source had no role in the design of this study, its execution, analyses, interpretation of the data, or decision to submit the results. Copyright: ( 2020 , American Society for Clinical Investigation.

Submitted: September 23, 2019; Accepted: January 22, 2020; Published: April 6, 2020.

Reference information: J Clin Invest. 2020;130(5):2364-2376. https://doi.org/10.1172/JCI133595. 


\section{Introduction}

Long-term renal allograft survival is limited for 2 reasons: first, conventional immunosuppression is not capable of preventing chronic rejection, which is responsible for more than half of graft losses; and second, conventional immunosuppressive therapy has serious side effects leading to increased morbidity and even death for patients with functioning allografts $(1,2)$. Thus, there is a great need for an antirejection therapy with fewer side effects and greater efficacy. The ideal therapy would involve donor-specific immunosuppression in the absence of systemic downregulation of the immune response.

Cellular therapies are gaining increasing attention as an alternative to conventional drug therapy for the control of allograft rejection (3). Effective rejection prophylaxis in the absence of immunosuppressive drugs has been achieved by establishing durable hematopoietic chimerism through combined or sequential hematopoietic stem cell application in kidney transplantation (4-7). The administration of hematopoietic stem cells, however, is an intervention that is limited by the toxicity of the conditioning regimen, the occurrence of graft-versus-host disease, and the need for a HLA-well-matched donor. Mixed chimerism is preferred to full donor chimerism as a means of inducing transplant tolerance because it offers greater safety (8). However, mixed donor chimerism has often been temporary, thus limiting its efficacy, but still associated with the risk of graft-versus-host disease (8). More recently, regulatory cell populations such as regulatory $\mathrm{T}$ lymphocytes (Tregs) have been evaluated for the prevention of kidney transplant rejection in preclinical models and are currently being tested in clinical trials (9).

We have developed a cell therapeutic involving modified immune cells (MICs) that, when injected into the recipient before transplantation, were capable of inducing donor-specific immunosuppression in various animal models such as rat heart and hind limb transplantation or pig kidney transplantation $(10,11)$. MICs were also effective in reversing a severe refractory rejection episode after allogeneic hematopoietic stem cell transplantation during emergency treatment in a pediatric patient (10).

In previous studies, we focused on the phenotypic and functional characterization of MICs. When monocytes were treated with the alkylating agent mitomycin $\mathrm{C}$ and then matured to DCs, the resulting cells showed the morphology and phenotype of early immature DCs and strongly suppressed the $\mathrm{T}$ cell response. FACS analysis revealed low expression of stimulatory molecules such as CD80, CD83, CD86, and HLA class II histocompatibility antigen $\gamma$ chain (HLA-DR) (12). When injected into the prospective experimental graft recipient, donor-derived MICs preferentially accumulated in peripheral lymphoid organs and induced regulatory lymphocytes (10).

Our aim was to translate the findings from preclinical experiments to a clinical phase I study in living donor kidney transplantation.

\section{Results}

Outcomes during the study phase. We conducted a 30-day singlearm, single-center phase I clinical trial to determine the safety and feasibility of intravenous administration of donor-derived MICs for individualized immunosuppression in living donor kidney transplant recipients. From August 2015 to February 2017, fourteen donor (D) and recipient (R) pairs were screened for inclusion in the TOL-1 study (Supplemental Figure 1; supplemental material available online with this article; https://doi.org/10.1172/ JCI133595DS1). Baseline characteristics of patients are shown in Table 1 and Supplemental Table 1. The MIC product was ultimately administered intravenously to 10 patients (R1-R7, R11, R12, R14) on the day of donor leukapheresis and product preparation as a 1-time administration. Patients received a prescribed dose of either $1.5 \times$ $10^{6}$ MICs per kg BW on day $-2\left(n=3\right.$, group A) or $1.5 \times 10^{8}$ MICs per $\mathrm{kg} \mathrm{BW}$ on day $-2(n=3$, group B) or day $-7(n=4$, group C) before living donor kidney transplantation, in addition to post-transplantation immunosuppression with cyclosporine A (CyA), entericcoated mycophenolate sodium (EC-MPS), and methylprednisolone. The TOL-1 study design is depicted in Figure 1.

Data collected during the first 30 days after transplantation showed that MIC infusions were extremely well tolerated. A total of 69 adverse events (AEs) including 3 serious AEs (SAEs) occurred in the 10 treated patients (Table 2 and Supplemental Tables 2 and $3)$. AEs were either unlikely to be related $(n=1)$ or not related $(n=68)$ to MIC therapy. During the study phase, no positive crossmatch results, de novo donor-specific HLA Abs (DSAs), or rejection episodes occurred, and all patients had stable kidney graft function with a serum creatinine level below $2 \mathrm{mg} / \mathrm{dL}$ on day 30 after surgery (Table 2, Supplemental Table 4, and Supplemental Figure 2).

No donor chimerism was detectable after MIC infusion. One day after MIC infusion, no donor chimerism was detectable in the 10 patients (Supplemental Table 5). An absence of donor chimerism was further confirmed on day -1 before and on days 7 and 30 after transplantation. As expected from chimerism analyses, none of the patients showed clinical evidence of graft-versus-host disease during follow-up.

Clinical outcomes up to 1 year after transplantation. After the study endpoint on day 30, patients were followed to day 360 after kidney transplantation. Immunosuppressive therapy in patients in group $\mathrm{C}$ was reduced by lowering the doses of CyA and EC-MPS and stopping corticosteroids (Supplemental Figure 3). During follow-up, no de novo DSAs or rejection episodes were noted, and all patients had stable kidney graft function (Table 3, Supplemental Table 4, and Supplemental Figure 2). On day 360 after surgery, the median serum creatinine was $1.4 \mathrm{mg} / \mathrm{dL}$ (range, 1.1-2.1 mg/dL), the median estimated glomerular filtration rate (eGFR) was 58 $\mathrm{mL} / \mathrm{min} / 1.73 \mathrm{~m}^{2}$ (range, $37-75 \mathrm{~mL} / \mathrm{min} / 1.73 \mathrm{~m}^{2}$ ), and the median urinary protein excretion was $10 \mathrm{~g} / \mathrm{moL}$ creatinine (range, $2-19 \mathrm{~g} /$ $\mathrm{moL})$. No opportunistic infections were noted. In particular, no BK virus $(\mathrm{BKV})$ replication was observed during rigorous post-transplantation screening. A total of 10 nonopportunistic infectious episodes occurred in 4 of the 10 patients (Table 3). No post-transplantation diabetes mellitus (PTDM), leukopenia, episodes of diarrhea, post-transplantation lymphoproliferative disease (PTLD), or other malignancies were observed. The total antihypertensive therapeutic intensity score (TIS) was lower on day 360 after transplantation than before surgery, particularly for patients in group C, and normal BP was maintained in all patients.

On the basis of previous experimental studies, we expected that treatment with the highest MIC cell numbers 7 days before transplantation would induce the strongest donor-specific immu- 


\section{Table 1. Patients' baseline characteristics}

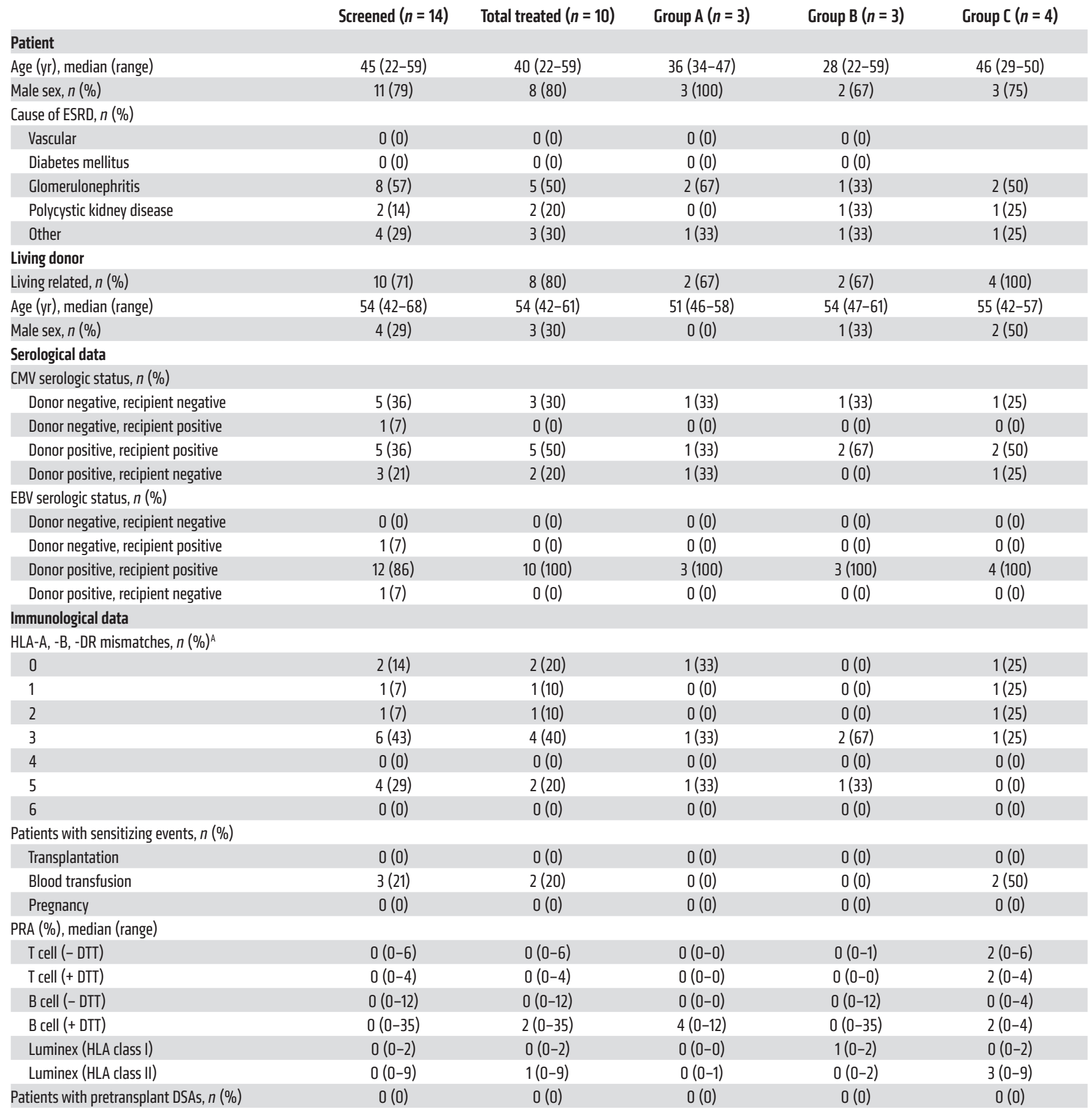

${ }^{A} \mathrm{HLA}$ mismatches for each treated patient are indicated in Supplemental Table 1. ESRD, end-stage renal disease.

nosuppression. Therefore, we performed detailed immunological testing on patients in group C (R7, R11, R12, and R14), whose MIC administration protocol corresponded to this schedule and who were on low immunosuppression.

Antidonor $T$ lymphocyte responses in MIC-treated patients. Patients in group $\mathrm{C}$ showed preserved lymphocyte proliferation in response to unspecific polyclonal stimulators on day 360 after transplantation compared with before MIC infusion, indicating an intact general immune response (Figure 2A). This was confirmed by allogeneic stimulation with third-party cells (Figure 2B). In contrast, the $\mathrm{T}$ cell response against the respective specific donor was absent on day 360 compared with what was observed before MIC infusion (Figure 2B). Five of 6 transplanted controls who were not treated with MICs showed normal Tlymphocyte reactivity to cells derived from their respective donors (Figure 2C), even though these patients were still on triple immunosuppressive therapy. 


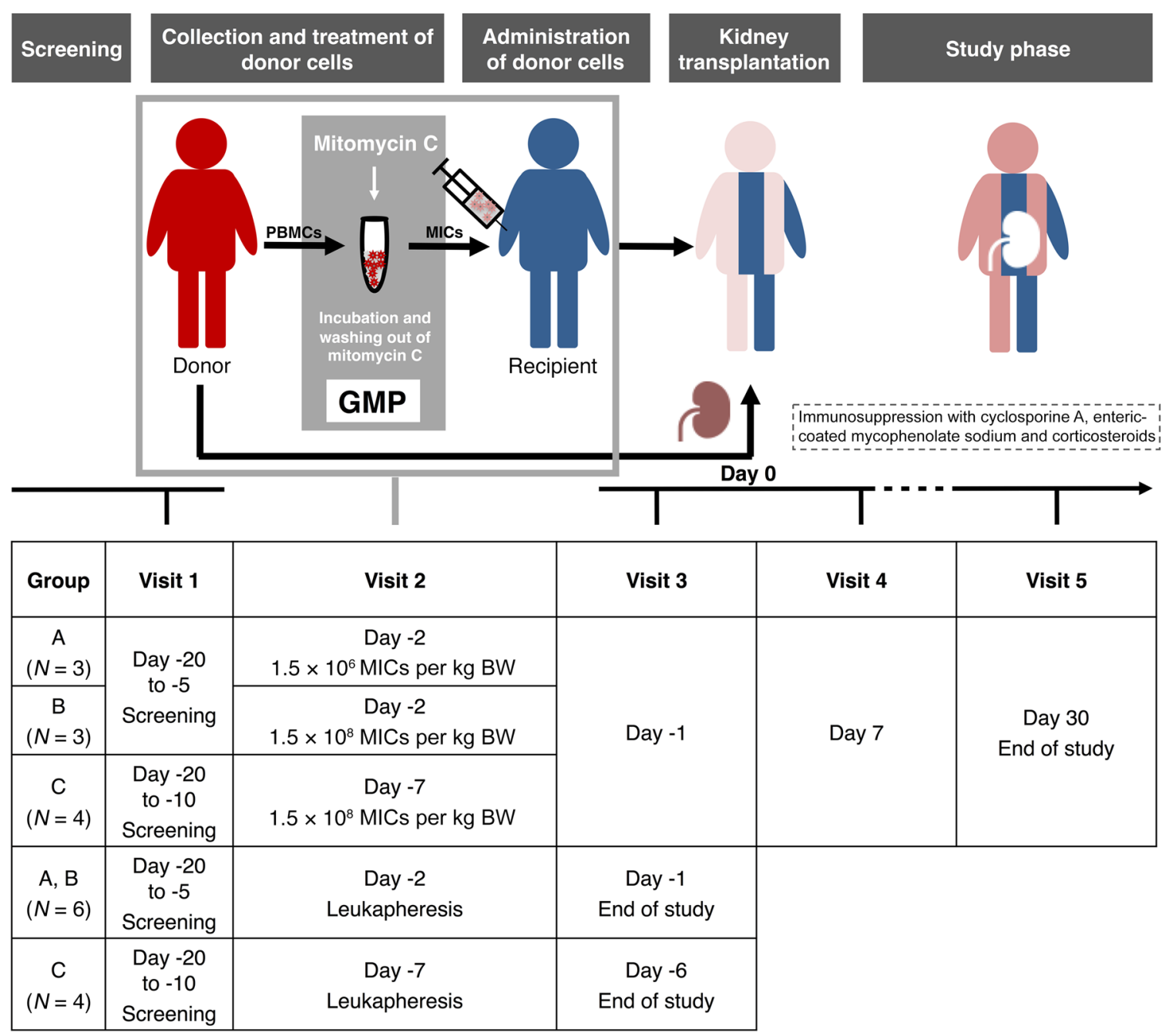

Figure 1. TOL-1 study design. On day -2 (groups A and B) or -7 (group C) before kidney transplantation, PBMCs were collected from donors by unstimulated standard leukapheresis. PBMCs were transferred to the good manufacturing practice (GMP) facility and incubated with the proliferation inhibitor mitomycin C. After washing out of mitomycin C and quality control, the final product (MICs) was administered to patients on the same day, approximately 12 hours after donor leukapheresis. In the intensive care unit, patients were intravenously administered either $1.5 \times 10^{6} \mathrm{MICs}$ per kg BW on day -2 ( $n=3$, group A) or $1.5 \times 10^{8}$ MICs per kg BW on day $-2(n=3$, group B) or day $-7(n=4$, group C) before living donor kidney transplantation. After transplantation, patients received immunosuppressive therapy with CyA, EC-MPS, and corticosteroids according to the center's protocol. The primary outcome measure was the frequency of AEs on day 30 (end of study).

Figure 2, D-G, shows the response of individual patients before (days $-7,-6$, and -1 ) and after transplantation (days 7,30 , $60,90,135,180,270$, and 360). Pretransplantation data include values before (day -7 ) and after MIC therapy (days -6 and -1 ). The measurement in patient $\mathrm{R} 7$ showed reduced responsiveness to the donor, with preserved responsiveness to third-party cells 1 day after MIC administration. This effect was maintained throughout follow-up (Figure 2D). In contrast, in patient R11, high proliferation in response to the donor was found 6 days after MIC therapy during an upper respiratory tract infection, and on day 180 after urinary tract infection (UTI) with severe allergic reaction to a quinolone antibiotic (Figure 2E). A similar reaction was found in patient R12 on day 180 after pneumonia and UTI (Figure 2F). In both patients, R11 and R12, the antidonor response disappeared completely during further follow-up, with a preserved responsiveness to third-party cells. Patient R14 had an increased response to the donor on day 90 (Figure $2 \mathrm{G}$ ). This was at the time of steroid withdrawal and 2 weeks before some deterioration of kidney graft function was observed. The kidney graft biopsy, however, showed no abnormality (Supplemental Table 4), and responsiveness to the original donor disappeared further in the clinical course, without additional treatment. Of note, from day 270 after transplantation onward, all patients showed a completely suppressed $\mathrm{T}$ lymphocyte response to the donor, with normal reactivity against third-party cells.

$H L A A b$ and Ab titers against bacterial and viral immunizations. During follow-up, no de novo DSAs were detected (Table 3). This finding raised the question of whether the memory B cell response to donor-unrelated antigens as induced by previous immunizations was also affected. Ab titers against measles (median, 4400 $\mathrm{mIU} / \mathrm{mL}$; range, 200-11,000 $\mathrm{mIU} / \mathrm{mL}$ ), mumps (median, 400; range, 230-8000), rubella (median, $41 \mathrm{IU} / \mathrm{mL}$; range, 9-160 IU/ 


\section{Table 2. Summary of primary and secondary outcome measures in 10 treated patients (TOL-1 study phase to day 30 )}

\begin{tabular}{|c|c|c|c|c|}
\hline & Total treated $(n=10)$ & Group A $(n=3)$ & Group B $(n=3)$ & Group C $(n=4)$ \\
\hline \multicolumn{5}{|l|}{ Primary outcome measure } \\
\hline $\mathrm{AEs}^{\mathrm{A}}$ (cumulative) & 69 & 16 & 24 & 29 \\
\hline Metabolism and nutrition disorders & 16 & 6 & 3 & 7 \\
\hline Gastrointestinal disorders & 19 & 2 & 8 & 9 \\
\hline Vascular disorders & 7 & 1 & 2 & 4 \\
\hline Other & 9 & 4 & 3 & 2 \\
\hline AEs per patient, median (range) & $7(3-10)$ & $6(3-7)$ & $7(7-10)$ & $8(3-10)$ \\
\hline \multicolumn{5}{|l|}{ Grade (1, mild; 2 , moderate; 3 , severe; 4, life-threatening; 5 , death) } \\
\hline 1 & 62 & 16 & 23 & 23 \\
\hline 2 & 7 & 0 & 1 & 6 \\
\hline \multicolumn{5}{|l|}{ Secondary outcome measures, $n(\%)$} \\
\hline Cumulative incidence of infection & $2(20)^{B}$ & $1(33)$ & $0(0)$ & $1(25)$ \\
\hline Cumulative incidence of CMV reactivation & $0(0)$ & $0(0)$ & $0(0)$ & $0(0)$ \\
\hline Cumulative incidence of PTLD & $0(0)$ & $0(0)$ & $0(0)$ & $0(0)$ \\
\hline Cumulative incidence of DGF (dialysis within first week) & $0(0)$ & $0(0)$ & $0(0)$ & $0(0)$ \\
\hline Development of positive CDC T, B, U cell crossmatch and/or ELISA crossmatch & $0(0)$ & $0(0)$ & $0(0)$ & $0(0)$ \\
\hline Development of donor-specific Abs & $0(0)$ & $0(0)$ & $0(0)$ & $0(0)$ \\
\hline Cumulative incidence of biopsy-proven cellular rejection (>BANFF borderline) & $0(0)$ & $0(0)$ & $0(0)$ & $0(0)$ \\
\hline Cumulative incidence of biopsy-proven Ab-mediated rejection & $0(0)$ & $0(0)$ & $0(0)$ & $0(0)$ \\
\hline Patients with stable graft function on day 30 (serum creatinine $<2 \mathrm{mg} / \mathrm{dL}$ ) & $10(100)$ & $3(100)$ & $3(100)$ & $4(100)$ \\
\hline Graft and patient survival on day 30 & $10(100)$ & $3(100)$ & $3(100)$ & $4(100)$ \\
\hline
\end{tabular}

${ }^{A}$ According to CTCAE, version 4.03. ${ }^{\mathrm{B} T w o}$ infectious complications were recorded, namely suspected central venous (CV) catheter-related infection (patient R2) and postoperative infection after re-surgery (patient R11). BANFF, Banff Classification of Renal Allograft Pathology.

$\mathrm{mL}$ ), varicella (median, $1350 \mathrm{mIU} / \mathrm{mL}$; range, 410-3500 mIU/ $\mathrm{mL}$ ), diphtheria (median, $0.17 \mathrm{IU} / \mathrm{mL}$; range, 0.04-0.33 IU/mL), and tetanus (median, $1.5 \mathrm{IU} / \mathrm{mL}$; range, 0.5-2.1 IU/mL) were lowest on day 30 after transplantation but reached pretransplantation levels during further follow-up (Supplemental Figure 4).

Numbers of $T$ lymphocytes, B lymphocytes, and NK cells. The numbers of $\mathrm{CD} 4^{+}$and $\mathrm{CD} 8^{+} \mathrm{T}$ lymphocytes as well as activated $\mathrm{CD}^{+}$and $\mathrm{CD}^{+}{ }^{+} \mathrm{T}$ lymphocytes remained stable before and after transplantation (Supplemental Figure 5, A-D). CD19+ B lymphocytes were highest on day 30 after surgery, with a median of 300/ $\mu \mathrm{L}$ (range, $149-561 / \mu \mathrm{L}$ ), but returned to pretransplantation levels on day 180 , with a median of $35 / \mu \mathrm{L}$ (range, 25-247/ $\mu \mathrm{L}$ ) (Supplemental Figure 5E). CD $16^{+} \mathrm{CD} 56^{+} \mathrm{NK}$ cells behaved inversely, as they were lowest on day 30 after transplantation, with a median of $60 / \mu \mathrm{L}$ (range, $33-73 / \mu \mathrm{L}$ ) but increased to a median of $104 / \mu \mathrm{L}$ (range, $93-154 / \mu \mathrm{L}$ ) on day 180 (Supplemental Figure $5 \mathrm{~F}$ ).

Unchanged Treg frequencies. The percentage of $\mathrm{CD} 4^{+} \mathrm{CD} 25^{+}$ FoxP3 ${ }^{+} \mathrm{CD} 127^{-}$Tregs was low on day 30 after transplantation (Figure 3) during the period of potent immunosuppressive therapy. The median percentage increased from $1 \%$ (range, $0 \%-1 \%$ ) on day 30 to $3 \%$ (range, $1 \%-5 \%$ ) on day 180 (Figure 3 C). This value was comparable to the pretransplantation and pretreatment level of $3 \%$ (range, $2 \%-4 \%$ ).
Strongly increased regulatory B lymphocyte frequencies. Interestingly, the percentage of $\mathrm{CD} 19^{+} \mathrm{CD} 24^{\mathrm{hi}} \mathrm{CD} 38^{\text {hi }}$ transitional B lymphocytes (regulatory B lymphocytes [Bregs]) was low until day 30 after transplantation, with a median of $2 \%$ (range, 0\%-6\%) (Figure $4 \mathrm{~B}$ ) but showed a striking increase thereafter. Breg percentages increased to a median of $20 \%$ (range, $5 \%-40 \%$ ) on day 180 , far exceeding the pretransplantation levels, which showed a median of only $6 \%$ (range, $0 \%-11 \%$ ) before MIC infusion. Increases were detected not only in the relative proportion but also the absolute Breg numbers, from a median of $4.5 / \mu \mathrm{L}$ (range, $0.3-15 / \mu \mathrm{L}$ ) on day 30 to $10 / \mu \mathrm{L}$ (range, $3.8-15 / \mu \mathrm{L}$ ) on day 180 (data not shown).

Breg percentages for group $\mathrm{C}$ patients in comparison with Breg percentages for transplanted controls without MIC infusion are shown in Figure 4C. Before MIC infusion, the values were comparable between patients in group $\mathrm{C}$ and transplanted controls, with a median of $6 \%$ and $11 \%$, respectively. In contrast, Breg percentages in MIC-treated patients dramatically increased after transplantation and were $4,3,9,19,26$, and 13 times higher than in transplanted controls on days 60, 90, 135, 180, 270, and 360 after transplantation, respectively. Patient R11, who was treated with high-dose methylprednisolone because of an allergic reaction to a quinolone antibiotic, had a lower percentage (5\%) of Bregs on day 180. Even this percentage was 5 times higher than that seen in 
Table 3. Outcomes and complications for 10 patients (TOL-1 study phase and follow-up out to day 360)

\begin{tabular}{|c|c|c|c|c|}
\hline & Total treated $(n=10)$ & Group A $(n=3)$ & Group B $(n=3)$ & Group C $(n=4)$ \\
\hline Biopsy-proven rejection ( $\geq$ BANFF IA), $n(\%)$ & $0(0)$ & $0(0)$ & $0(0)$ & $0(0)$ \\
\hline Development of de novo DSAs (A, B, DR, DQ), $n(\%)$ & $0(0)$ & $0(0)$ & $0(0)$ & $0(0)$ \\
\hline Patients with opportunistic infections, $n(\%)$ & $0(0)$ & $0(0)$ & $0(0)$ & $0(0)$ \\
\hline Pneumonia & 0 & 0 & 0 & 0 \\
\hline CMV reactivation $>1000$ copies $/ \mathrm{mL}$ & 0 & 0 & 0 & 0 \\
\hline Other infection & 0 & 0 & 0 & 0 \\
\hline Patients with nonopportunistic infections, $n$ (\%) & $4(40)$ & $1(33)$ & $0(0)$ & $3(75)$ \\
\hline Infectious episodes, $n$ & 10 & 1 & 0 & 9 \\
\hline CV-associated infection & 1 & 1 & 0 & 0 \\
\hline UTI & 6 & 0 & 0 & 6 \\
\hline \multicolumn{5}{|l|}{ BP on day 360 , median (range) } \\
\hline Systolic BP (mmHg) & $125(110-145)$ & $130(110-145)$ & $120(120-130)$ & $129(120-140)$ \\
\hline Total antihypertensive TIS & $0.75(0.13-2.5)$ & $0.75(0.5-1.5)$ & $1.5(0.13-1.5)$ & $0.75(0.17-2.5)$ \\
\hline Change of total antihypertensive TIS from baseline & $-0.22(-4.63-0.75)$ & $-0.25(-2.25-0)$ & $0(-0.19-0.75)$ & $-1.04(-4.63-0)$ \\
\hline PTDM, $n(\%)$ & $0(0)$ & $0(0)$ & $0(0)$ & $0(0)$ \\
\hline Leukopenia <3.5/nL, $n(\%)$ & $0(0)$ & $0(0)$ & $0(0)$ & $0(0)$ \\
\hline Documented diarrhea, $n(\%)$ & $0(0)$ & $0(0)$ & $0(0)$ & $0(0)$ \\
\hline Patients with surgical complications ${ }^{\mathrm{A}}, n(\%)$ & $4(40)$ & $0(0)$ & $2(67)$ & $2(50)$ \\
\hline Bleeding & $1(10)$ & $0(0)$ & $0(0)$ & $1(25)$ \\
\hline Wound-healing disturbances & $2(20)$ & $0(0)$ & $2(67)$ & $0(0)$ \\
\hline Urinary leakage & $1(10)$ & $0(0)$ & $0(0)$ & $1(25)$ \\
\hline
\end{tabular}

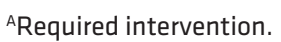

the transplanted controls (day 180: median, 1\%; range, 0\%-2\%). In this patient, Bregs increased subsequently to reach $13 \%$ on day 720 (Supplemental Figure 6).

To further exclude a decisive effect of steroids on the observed differences in Breg percentages, we compared MIC-treated patients in group $\mathrm{C}$ with a second independent cohort of transplanted controls without MIC infusion, who were stratified according to methylprednisolone dose (Figure 4D). Bregs in group C patients were 68 and 44 times higher than those in transplanted control patients without steroid treatment 180 and 360 days after transplantation, respectively. At last follow-up (median, 810 days; range, 720-1080 days) after transplantation and more than 1.5 years after steroid withdrawal in all MIC-treated patients in group C, Breg percentages represented a median of $7 \%$ (range, $5 \%-11 \%$ ) and were 24 times higher than in transplanted controls who did not receive steroid treatment. The percentage of Bregs was also higher than the $2 \%$ Bregs detected in a control patient with operational tolerance from our center (Figure 4D and Supplemental Figure 6).

It was of particular interest whether Bregs also increased in patients in group A (receiving a reduced cell dose on day-2) and group B (receiving the full cell dose on day -2). For this purpose, we analyzed frozen samples of Bregs from patients in groups A, $\mathrm{B}$, and $\mathrm{C}$. As expected from preclinical experiments, patients in group $\mathrm{C}$ showed the highest Breg percentages, exceeding the values for the patients in groups A and B by a factor of 68 and 20, respectively, on day 180 after transplantation (Figure 4E).

As shown in Figure 4F, the majority of Bregs from patients in group C produced the immunosuppressive cytokine IL-10 (median, 44\%-100\%).

High levels of immunosuppressive cytokines IL-10 and TGF- $\beta 1$. Supplemental Tables 6 and 7 list 20 serum proteins with the largest decreases or increases in all 10 patients after (day-1) as compared with before (day-2 or -7) MIC administration. The most intriguing findings were the strong decrease in HLA class II histocompatibility antigen and the increases in IL-10 and TGF- $\beta 1$ (Supplemental Tables 6 and 7 and Figure 5).

The same analysis on day 180 after transplantation compared with before MIC administration is shown in Supplemental Tables 8 and 9. The most important finding was that high levels of the immunosuppressive cytokines IL-10 and TGF- $\beta 1$ were maintained, reaching a median of $1.1 \mathrm{pg} / \mathrm{mL}$ (range, $0.0-1.8 \mathrm{pg} / \mathrm{mL}$ ) and $9107 \mathrm{pg} / \mathrm{mL}$ (range, 7602-19,237 pg/mL), respectively (Supplemental Figure 7).

Ingenuity pathway analysis (IPA) of proteome data after versus before MIC administration pointed to 23 biological functions that were significantly upregulated and 2 that were downregulat- 
A

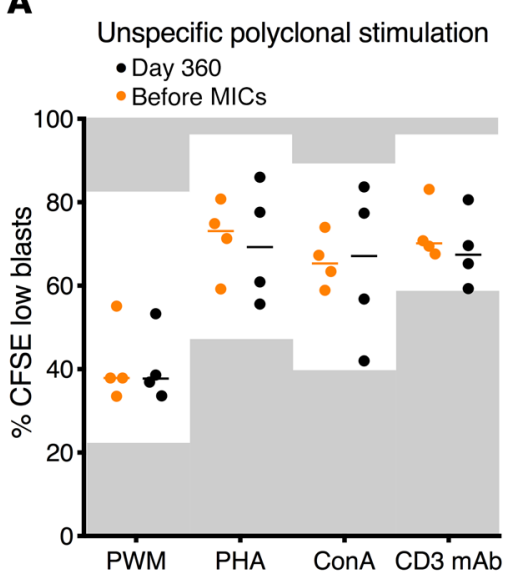

D

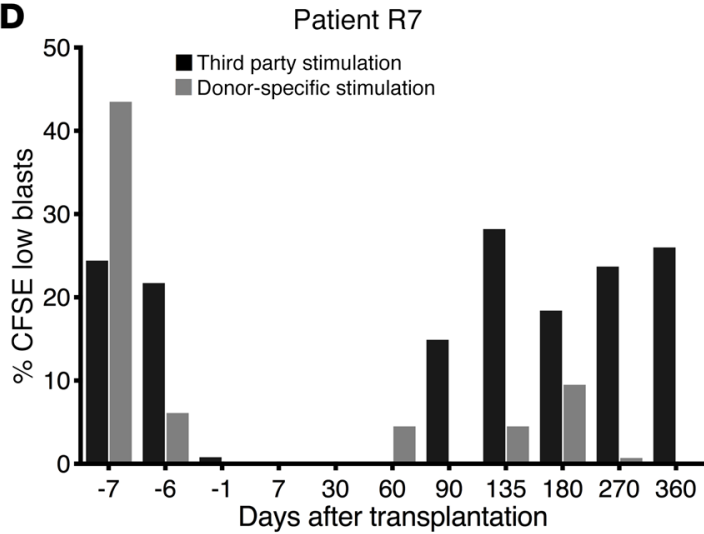

F

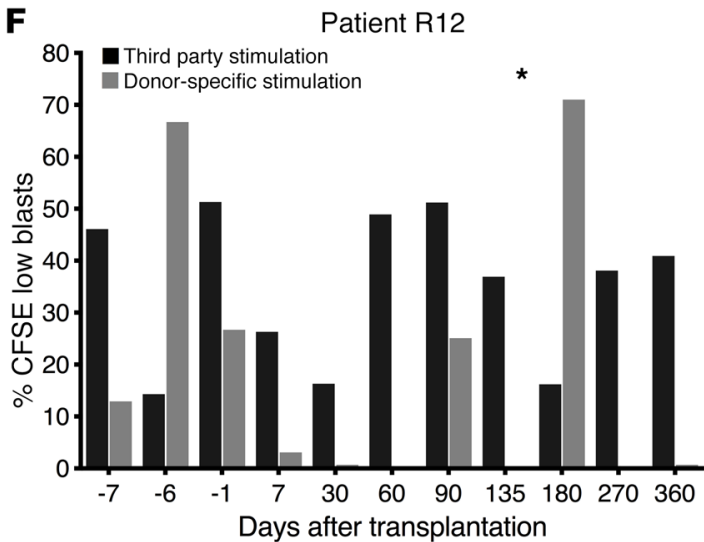

Third party and donor-specific stimulation - Day 360

- Before MICs

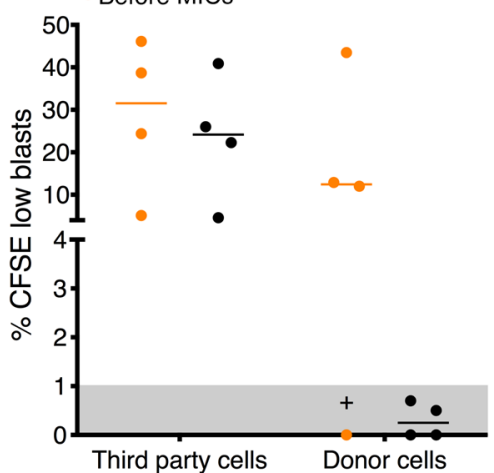

E

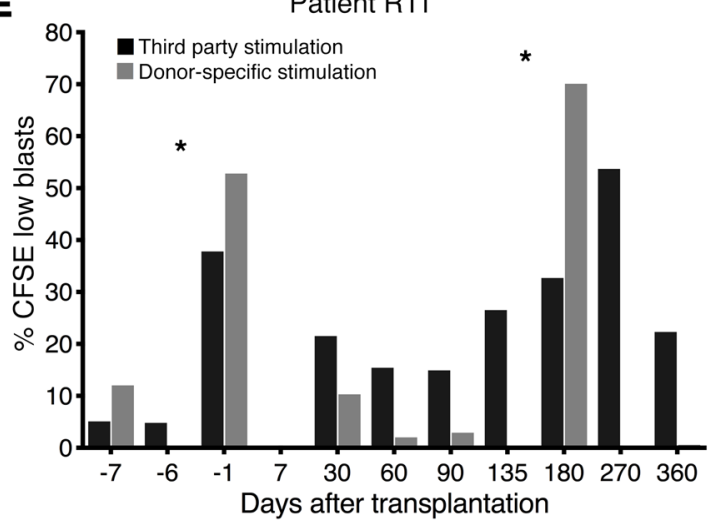

G

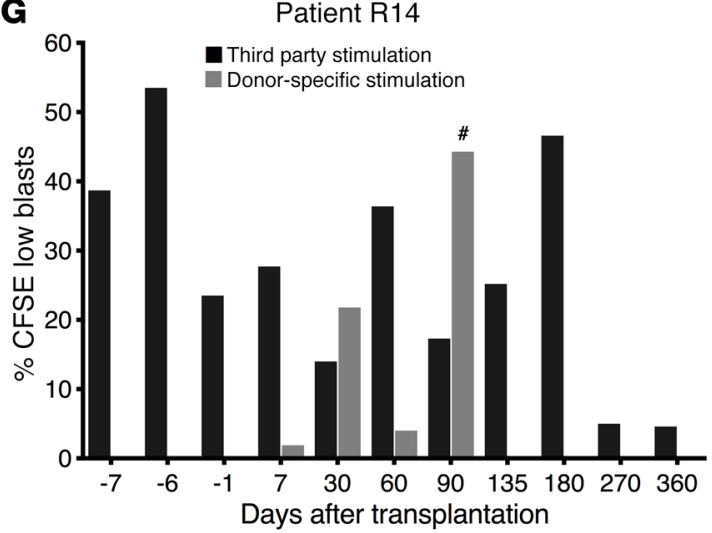

Figure 2. Polyclonal, third-party, and donor-specific lymphocyte response of MIC-treated patients in vitro. (A) Polyclonal stimulation (pokeweed mitogen [PWM], phytohemagglutinin [PHA], concanavalin A [ConA], CD3 mAb) of blood lymphocytes from patients in group C (R7, R11, R12, R14) on day 360 (black circles) versus before MIC treatment on day -7 (orange circles). Individual measurements and the median are shown. Values outside the normal range for healthy individuals are highlighted in gray. Patients showed preserved in vitro lymphocyte proliferation after transplantation. (B and C) Thirdparty and donor-specific stimulation of blood lymphocytes from patients in group $[$ on day 360 (black circles) versus before MIC treatment on day -7 (orange circles) (B), or versus transplanted controls on triple immunosuppressive therapy (red circles) (C). Individual measurements and the median are shown. Values outside the normal range for healthy individuals are highlighted in gray. The findings indicate preserved immunological responsiveness of recipient T lymphocytes against irradiated third-party cells, with reduced responsiveness to donor cells after transplantation compared with before transplantation and MIC infusion (B). Transplanted control patients without MIC treatment showed higher responsiveness compared with MIC-treated patients on day 360 (C). (D-C) Third-party (black bars) and donor-specific (gray bars) stimulation of blood lymphocytes from individual patients in group C (R7, R11, R12, R14) was performed before (days $-7,-6$, and -1) and after transplantation (days 7, 30, 60, 90, 135, 180, 270, and 360), as well as before (day -7) and after (days -6 and -1) MIC infusion. In all experiments, stimulatory cells consisted of irradiated allogeneic PBMCs. T lymphocyte proliferation was assessed by CFSE staining. Plus sign indicates patient R14, who had no HLA-A, -B, or -DR mismatches with the donor; asterisk indicates infectious/inflammatory episodes; pound sign indicates at the time of steroid withdrawal. 
A
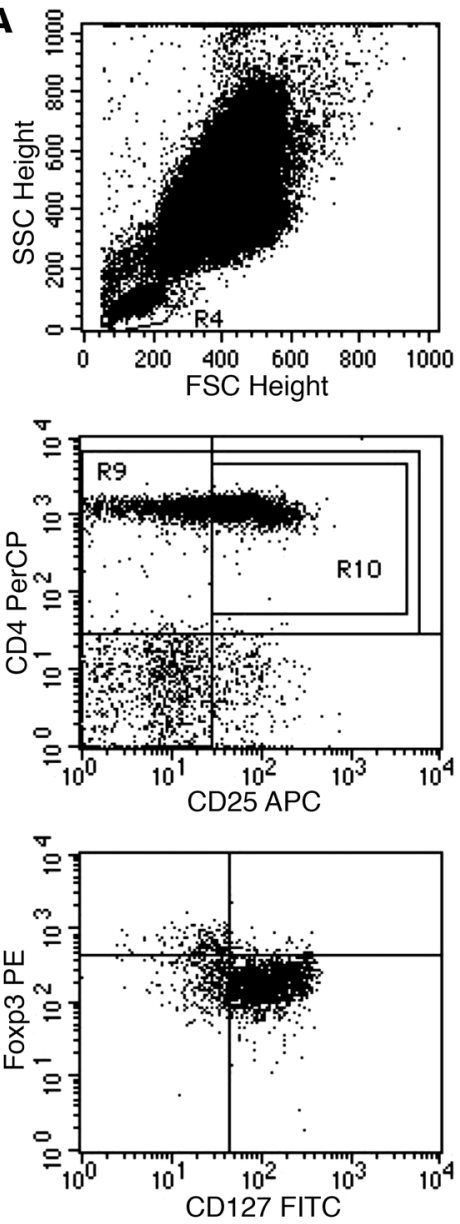

B Regulatory $T$ lymphocytes, MIC patients, fresh cells

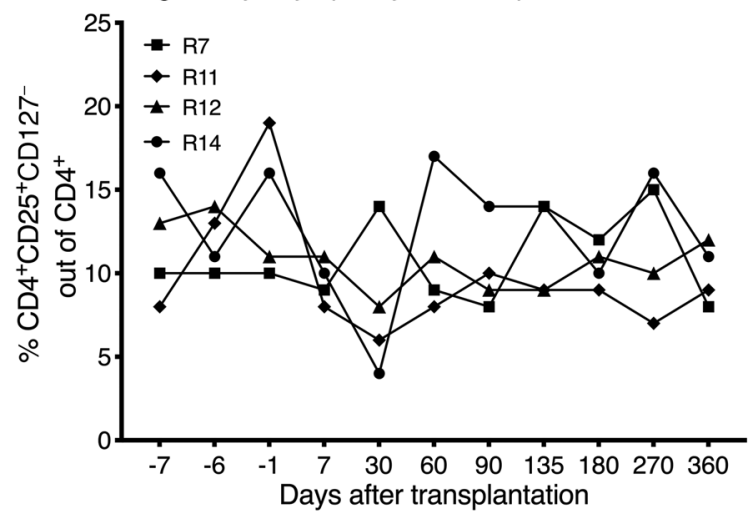

C

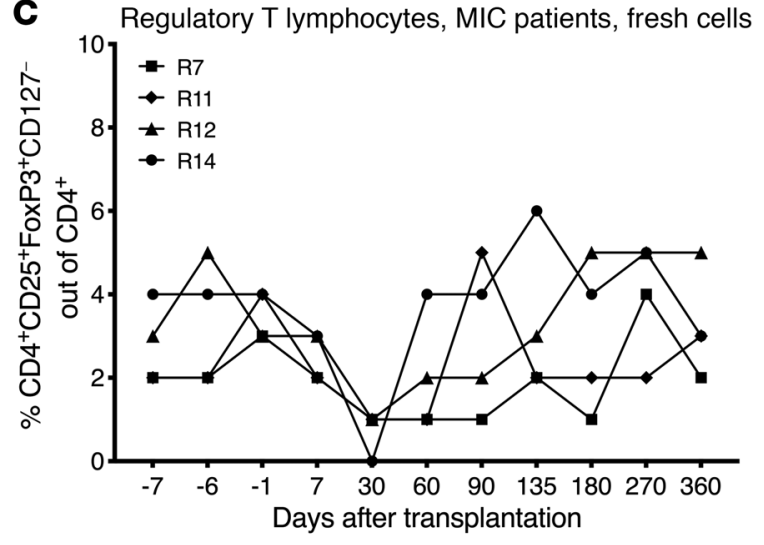

Figure 3. Tregs in MIC-treated patients. (A) Peripheral blood lymphocytes (PBLs) were gated in a forward scatter/side scatter (FSC/SSC) dot plot (gate R4). CD4+CD25+ PBLs were determined using a CD4/CD25 dot plot (R10) and further analyzed for coexpression of both $\mathrm{Foxp}^{+}$ and $\mathrm{CD} 127^{-}$or only $\mathrm{CD} 127^{-}$in a Foxp3/CD127 dot plot (CD4+CD25+ Foxp3 ${ }^{+}{ }^{+} D 127^{-}$and $\mathrm{CD}^{+}{ }^{+} \mathrm{CD} 25^{+} \mathrm{CD} 127-$ Treg subsets). APC, allophycocyanin; $\mathrm{PE}$, phycoerythrin; PerCP, peridininchlorophyll-protein. Individual measurements for the percentage of $\mathrm{CD} 4^{+} \mathrm{CD} 25^{+} \mathrm{CD} 127^{-}$Tregs (B) and $\mathrm{CD}^{+}{ }^{+} \mathrm{CD} 25^{+}{ }^{+}$oxP3 $^{+}$CD127- Tregs (C) in patients R7, R11, R12, and R14 from day -7 to day 360 are shown. Tregs were lowest on day 30 after kidney transplantation at the time of powerful immunosuppressive therapy. ed. Of the downregulated functions, suppression of immunological disease/hypersensitive reaction was of special interest in the context of transplantation (Figure 5).

Patients display an Immune Tolerance Network operational tolerance signature after MIC infusion. Since IL-10-producing Bregs were associated with operational tolerance, the Immune Tolerance Network operational tolerance signature was assessed for patients in group $\mathrm{C}$ and compared with that of patients in groups $\mathrm{A}$ and $\mathrm{B}$, who lacked the remarkable increase in Breg frequencies. Although some overlap with the other groups was observed, the strongest expression of IGKV4-1 and IGKV1D-13 on day 180 was found in group C patients (Supplemental Figure 8).

\section{Discussion}

The primary objective of this phase I clinical trial was to establish nontoxicity. MIC infusions were extremely well tolerated, with no infusion-related side effects. None of the 10 patients developed de novo DSAs or had rejection episodes, even though they received up to $1.3 \times 10^{10}$ allogeneic donor mononuclear cells before transplantation.

Since some of the results went beyond a classical safety study, we were encouraged to examine these data with regard to a possible efficacy of MIC therapy. Naturally, definitive conclusions cannot be drawn at this time. Group $C$ patients received the highest cell dose 7 days before surgery and were on low immunosuppression therapy during follow-up. They showed an absence of post-transplant cellular stimulation reactivity when tested against their respective kidney donors, whereas reactivity against third-party cells was retained. These patients also showed strong Breg induction with concomitant IL-10 production as well as evidence of an immune tolerance signature according to the definition of the Immune Tolerance Network. Taken together with the results obtained in our previous experimental studies, these data support the idea that donor-specific immunosuppression is induced by MIC treatment (10-12).

It is interesting that common infections, like upper respiratory tract infection or UTI, triggered a transient donor-specific responsiveness that disappeared again after resolution of the infection. From day 270 onward to the most recent post-transplantation follow-up (median, 810 days; range, 720-1080 days), all patients showed continued complete suppression of T lymphocyte responses to the specific donor's cells (data not shown).

An exciting observation in the MIC-treated patients in group $\mathrm{C}$ was the strong increase in $\mathrm{CD} 19^{+} \mathrm{CD} 24^{\mathrm{hi}} \mathrm{CD} 38^{\mathrm{hi}}$ transitional $\mathrm{B}$ lymphocytes (Bregs) after transplantation. Transitional CD19+ $\mathrm{B}$ cells, characterized by surface expression of CD24 ${ }^{\text {hi }} C D 38^{\text {hi }}$, have been proposed to be tolerance markers, given the data supporting their role in maintaining long-term allograft acceptance (13-18). Preserved levels of $\mathrm{CD} 19^{+} \mathrm{CD} 24^{\text {hi }} \mathrm{CD} 38^{\text {hi }}$ transitional B lymphocytes were described in operationally tolerant patients in a range of $4 \%-7 \%$ (14). Tebbe et al. found that in patients who received routine doses of the immunosuppressive agent CyA as a calcineurin inhibitor, the frequencies of $\mathrm{CD} 19^{+} \mathrm{CD} 24^{\text {hi }} \mathrm{CD} 38^{\text {hi }}$ transitional $\mathrm{B}$ 
A

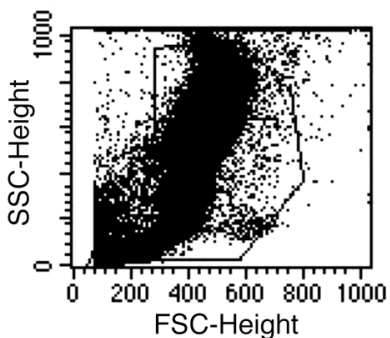

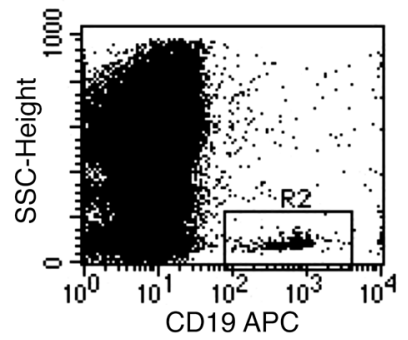

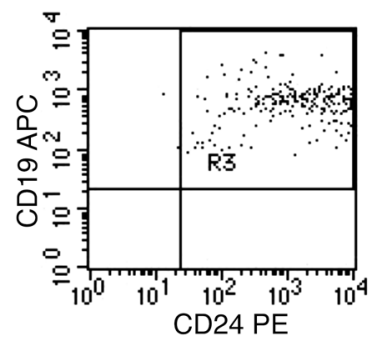

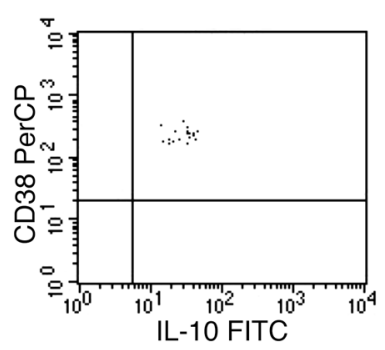
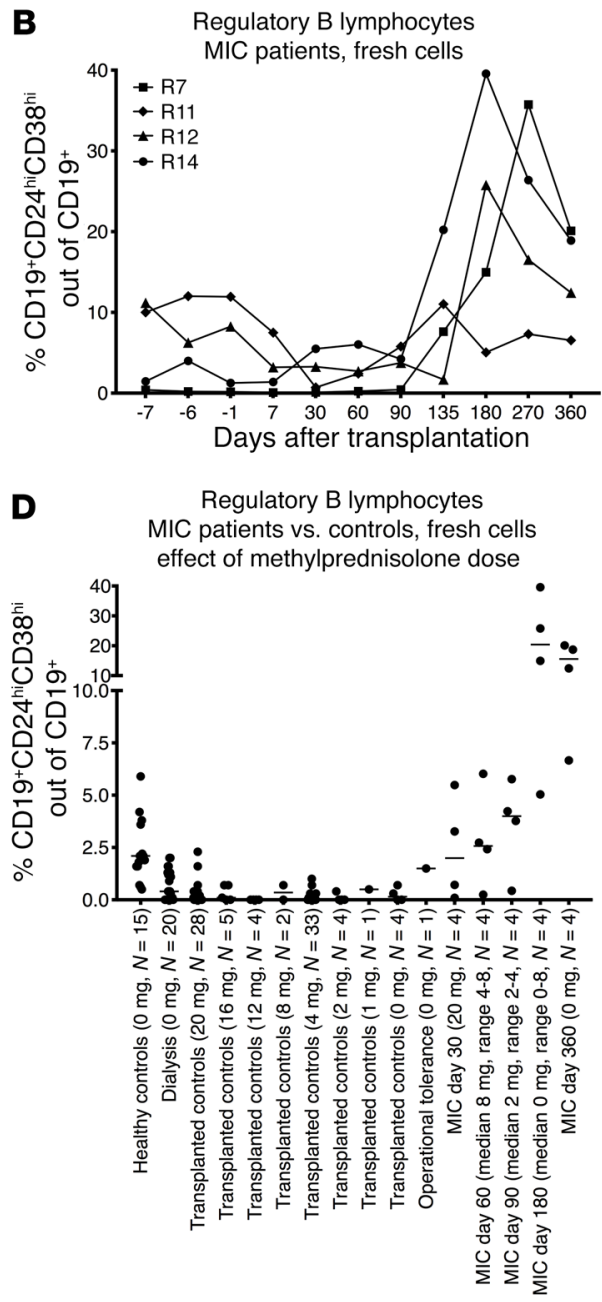

C

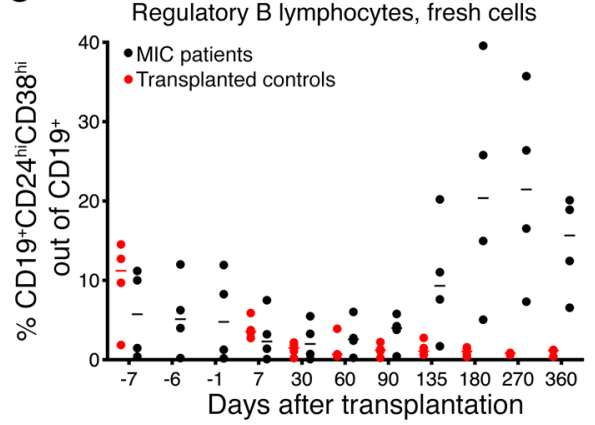

E
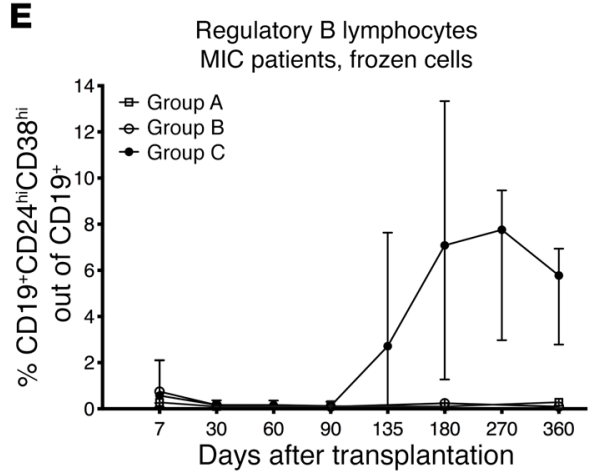

F IL-10-producing regulatory B lymphocytes cumulative post-transplant data in individual patients, frozen cells

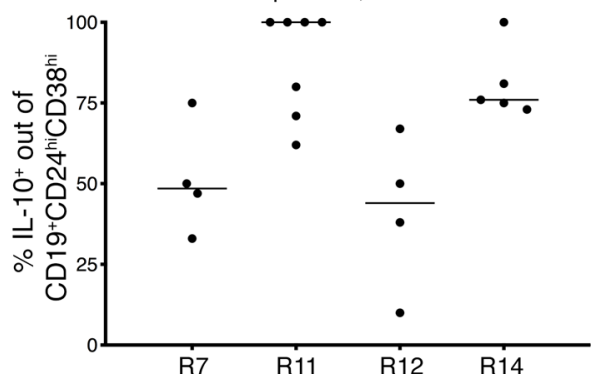

Figure 4. Bregs in MIC-treated patients compared with Bregs in transplanted controls. (A) PBLs were gated in a FSC/ SSC dot plot (gate R1). B lymphocytes were gated using a CD19/SSC gate (gate R2). CD19+CD24+ cells were determined (gate R3) within the CD19+ B lymphocytes (gate R2). On the basis of gate R3, CD24 $4^{\text {hi }} C D 38^{\text {hi }} B$ lymphocytes were analyzed (gate R6) and assigned as CD19+ CD24 ${ }^{\text {hi } C D 38}{ }^{\text {hi }}$ transitional B lymphocytes (Bregs). IL-10 production of CD19+CD24 ${ }^{\mathrm{hi}}$ CD38 hi transitional B lymphocytes was further investigated using a CD38/IL-10 gate based on gate R6. (B) Individual measurements of the percentage of CD19+ $C D 24^{\text {hi }}$ CD38 $8^{\text {hi }}$ Bregs in patients R7, R11, R12, and R14 from day -7 to day 360 . CD19+CD24 ${ }^{\text {hi }}$ CD38 ${ }^{\text {hi }}$ Bregs were low out to day 30 after kidney transplantation and increased out to day 180. (C) Individual measurements of the percentage of $\mathrm{CD}^{+}{ }^{+} \mathrm{CD} 24^{\mathrm{hi}} \mathrm{CD} 38^{\text {hi }}$ Bregs in patients in group C (black circles) were compared with measurements in transplanted controls (red circles). Individual measurements and the median are shown. Compared with transplanted controls, Breg percentages were 4, 3, 9, 19, 26, and 13 times higher in patients in group C on days $60,90,135,180,270$, and 360 after transplantation, respectively. (D) Individual measurements of the percentage of $\mathrm{CD} 19+\mathrm{CD} 24^{\text {hi }} \mathrm{CD} 38^{\text {hi }}$ Bregs in patients in group $C$ were compared with measurements in a second independent cohort of transplanted controls stratified according to steroid dose (shown in parentheses). Individual measurements and the median are shown. Compared with transplanted controls without steroid treatment, Breg percentages were 68 and 44 times higher in patients in group $C$ on days 180 and 360 after transplantation, respectively. (E) Percentage of CD19+CD24 ${ }^{\text {hi }}$ CD38 ${ }^{\text {hi }}$ Bregs in frozen cells from patients in groups A-C. The median and interquartile range are shown. In contrast to the percentage of CD19+CD24 ${ }^{\text {hi }} C D 38^{\text {hi }}$ Bregs from patients in groups $B$ and $C$, the percentage of Bregs for patients in group $A$ were 68 and 20 times higher, respectively, on day 180 after transplantation. (F) Percentage of IL-10-producing Bregs. Individual measurements and the median are shown for cumulative post-transplantation data for patients in group C. The majority of Bregs were producing the immunosuppressive cytokine IL-10. 


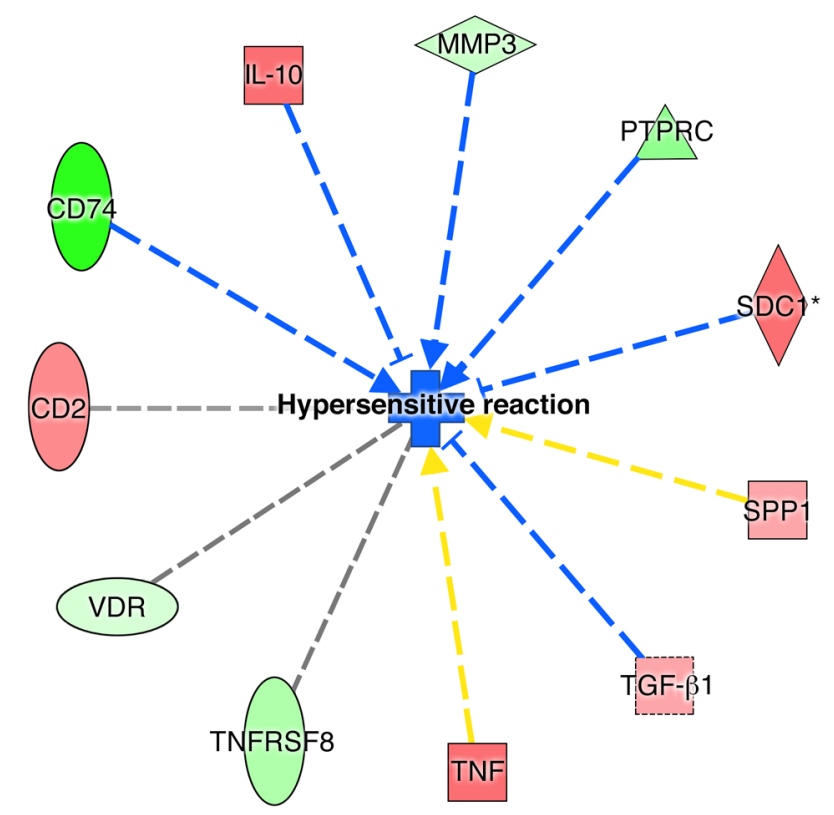

lymphocytes were in the range of $0 \%-5 \%$ during the first year after transplantation. Those patients who showed frequencies greater than $1 \%$ experienced no rejection episodes (17). In our study, the Breg frequencies in transplanted controls who were not subjected to MIC conditioning were comparable to those described by Tebbe et al., whereas the MIC-treated patients in group C had Breg frequencies in the range of 5\%-40\% (median, 20\%) on post-transplantation day 180, far exceeding the frequencies detected in the transplanted controls (day 180 median, 1\%) and in these group C patients before transplantation (day -7 median, $6 \%$ ).

Bregs modulate the effector function of both adaptive and innate immune cells (19). The suppressive function of Bregs is thought to be mediated in part by IL-10 (20). We found that the great majority of Bregs in the MIC-treated group C patients produced IL-10. This finding was further substantiated by the observation that IL-10 levels in the sera of these patients were increased.

Recent data suggest that the type and dose of immunosuppressive therapy may interfere with the induction of $\mathrm{CD} 19^{+} \mathrm{CD} 24^{\mathrm{hi}}$ CD38 ${ }^{\text {hi }}$ transitional B lymphocytes (Bregs) $(21,22)$. In a crosssectional study of 117 transplant recipients, Bottomley et al. found no significant association between steroids and Bregs (21), whereas in the Genetic Analysis of Molecular Biomarkers of Immunological Tolerance (GAMBIT) study, Rebollo-Mesa et al. observed slightly higher Breg percentages in patients with reduced or no steroids (22). To address this issue, we identified transplanted control patients on immunosuppressive therapy comparable to that for MIC-treated patients and analyzed their Breg frequencies retrospectively. Impressively, on day 135 after transplantation and before steroids were stopped in 3 of 4 group C patients, we found that Breg percentages were already 9 -fold higher than those in the transplanted controls. At last follow-up, more than 1.5 years after steroid withdrawal in all MIC-treated group C patients, Breg frequencies were 24-fold higher than in a second independent cohort of transplanted controls without steroid treatment, and considerably higher than the Breg percentages in a control patient with operational tolerance from our center. Data that also argue against an overriding interference
Figure 5. Immune reaction patterns induced by MIC therapy based on proteome analysis. IPA of proteome data on day -1 (after MIC infusion) versus days -2 and -7 (before MIC infusion) indicated significant downregulation of the biological function immunological disease/hypersensitive reaction after MIC administration but before kidney transplantation and application of immunosuppressive therapy. Upregulation (red shapes); downregulation (green shapes); predicted inhibition (blue shape); leads to inhibition (blue dashed lines); findings inconsistent (yellow dashed lines); findings not predicted (gray dashed lines) . CD74, HLA-DR; MMP3, stromelysin-1; PTPRC, receptor-type tyrosine-protein phosphatase C; SDC1*, syndecan-1; SPP1, osteopontin; TNFRSF8, TNF receptor superfamily member 8 (CD30); VDR, vitamin D3 receptor; CD2, T cell surface antigen CD2.

of immunosuppressive medication were derived from our current studies in a murine model of lupus nephritis. MIC-treated animals showed significantly higher Breg $\left(\mathrm{CD} 19^{+} \mathrm{CD} 5^{+} \mathrm{CD} 1 \mathrm{~d}^{\text {hi }}\right)$ frequencies than did untreated controls in the absence of any immunosuppressive therapy (our unpublished observations).

Studies on $\mathrm{CD}_{3} 4^{+}$stem cell transplantation reported donorspecific unresponsiveness when persistent chimerism was established $(23,24)$. In HLA-identical living donor kidney transplant recipients, complete withdrawal of immunosuppressive therapy was feasible without allograft rejection, even when chimerism was lost further along in the course of treatment (25). We learned from our preclinical studies that long-lasting chimerism was not established after MIC treatment. After infusion, MICs immediately left the circulation and preferentially accumulated in the spleens of treated rats, where the cells were detectable for a maximum of 7 days (our unpublished observations). Impressively, when PBMCs from MIC-treated animals were later transferred into naive rats, those rats tolerated heart allografts from the original MIC donor strain but not from a third party (10). This indicates the induction of regulatory cell populations in treated animals during the short contact of the recipient's immune system with donor MICs. In line with these observations, MIC-treated group C patients established no donor chimerism at a $1 \%$ detection limit of the assay, whereas Bregs were detectable at high percentages throughout the follow-up period.

A limitation of this study is the absence of surveillance biopsies beyond day 7 after transplantation to rule out alloimmune injury such as subclinical $\mathrm{T}$ cell-mediated inflammation. CyA was chosen rather than tacrolimus, because it represented the standard calcineurin inhibitor for unsensitized kidney recipients in our center at the time of the study, and since no interference of CyA with MIC treatment was found during preclinical experiments (26). We speculate that tacrolimus would provide similar results. In favor of this assumption are the results of a recent study showing that the Breg levels in stable immunosuppressed patients on tacrolimus were even higher than the levels in patients on CyA (17). We 
recognize the limitations of the data obtained in a phase I clinical trial and particularly in this trial, in which only 4 patients received the full MIC dose on day -7. Clearly, the observations described in this report are preliminary. Nevertheless, their exciting nature motivated us to submit the results at this time, because we believe they will stimulate immune tolerance research that builds on these preliminary observations.

In summary, MIC therapy was well tolerated in this phase I clinical trial and did not lead to humoral sensitization or rejections. The in vitro findings of an absence of specific cell-stimulatory reactivity against donor cells after transplantation, paralleled by a rise in Breg numbers and IL-10 levels, led us to hypothesize that MIC conditioning may constitute a promising method for inducing donor-specific immunosuppression in renal transplantation. Although our findings are promising, it is much too early to know whether this treatment is sufficiently tolerogenic to allow complete withdrawal of immunosuppression. We believe, however, that the preliminary phase I results are sufficiently encouraging to proceed with phase II and III clinical trials.

\section{Methods}

Study protocol. We performed a 30-day, single-arm, single-center phase I clinical trial to determine the safety and feasibility of intravenous administration of donor-derived MICs for individualized immunosuppression in living donor kidney transplant recipients (TOL-1 study).

The primary outcome measure was the safety and feasibility of intravenous administration of MICs as measured by the frequency of AEs in patients with stage 4 or 5 chronic kidney disease (i.e., GFR $<30$ $\mathrm{mL} / \mathrm{min} / 1.73 \mathrm{~m}^{2}$ ), who received a kidney transplant from a living donor. AEs were recorded according to Common Terminology Criteria for Adverse Events (CTCAEs), version 4.03. Secondary outcome measures included the incidence of infection, CMV reactivation, PTLD, delayed graft function (DGF), a positive complement-dependent cytotoxicity (CDC) T-, B-, unseparated-cell crossmatch and/or ELISA crossmatch result, development of DSAs, biopsy-proven cellular or Ab-mediated rejection, stable graft function, and graft and patient survival out to day 30 after transplantation. In addition, a predefined scientific program was implemented to investigate the possible efficacy of the therapy, including FACS analysis of lymphocyte subpopulations, Tregs, and Bregs, determination of serum cytokine levels, mixed lymphocyte reaction, and proteome and gene expression analyses. Patients were eligible for participation in the study if they had stage 4 or 5 chronic kidney disease in preparation for a first kidney transplant from a living donor aged 18 years or older. Only recipients of an $\mathrm{ABO}$-compatible transplant with a CDC panel-reactive Ab (PRA) of less than 20\%, no DSAs, and a negative CDC and ELISA crossmatch result with their respective donor on triple-drug immunosuppressive therapy with CyA, EC-MPS, and methylprednisolone were included. Female patients with child-bearing potential were on adequate contraception. All patients provided written informed consent. The main exclusion criteria were as follows: a psychiatric disorder, heart failure (New York Heart Association [NYHA] III or IV), severe liver disease, active hepatitis B or C or HIV infection, active bacterial, fungal, or viral disease, malignancy or malignancy in the 5 years prior to the screening, preexisting immunosuppression, vaccination with a live vaccine in the 3 months before screening, s/p splenectomy, substance abuse, pregnancy or lactation, female patients with possible HLA sensitization due to a pregnancy by or a child with the intended donor, allergy to the investigational drug or components of it, and other diseases that, in the opinion of the investigator, prohibited participation in the study or participation in another interventional study.

From August 2015 to February 2017, 14 donor and recipient pairs were screened for inclusion in the TOL-1 study (Supplemental Figure 1). A total of 12 donors underwent leukapheresis. The MIC product was given intravenously to 10 patients (R1-R7, R11, R12, and R14) on the day of donor leukapheresis and product preparation as a one-time administration. Patients were excluded for the following reasons: patient R8 was found to have a dissection of the right iliac and femoral artery during the pre-transplant CT scan evaluation. The evaluation had already been performed before screening, but dissection became obvious only after screening. In patient R9, acute hyperthyroidism was diagnosed during the second visit. Transplantation surgery had to be postponed in both patients. During screening, patient R10 was found to have a positive IgM XM-ONE crossmatch (Olerup) result, and patient R13 was found to have irregular warm-reacting Abs at the second visit. Though these were not formal contraindications for inclusion in the study, we decided not to perform cell therapy in either patient for safety reasons. Transplantation surgery was performed as scheduled but outside the TOL-1 study. Two donors (D8 and D10) were screened but received no leukapheresis for cell harvesting, since the corresponding recipients were excluded from the study. Leukapheresis was performed in donor D9 and D13, but cells were not further processed and were discarded for the reasons outlined above. For these donors, the study was finished according to the protocol with visit 3, on the day after leukapheresis.

Patients were treated sequentially in a stepwise approach with dose escalation from group A $\left(1.5 \times 10^{6}\right.$ MIC per kg BW) to group B $\left(1.5 \times 10^{8}\right.$ MICs per kg BW) to account for suspected possible AEs related to the MIC product such as embolism, inflammation, allergy, or an $\mathrm{AE}$ due to mitomycin $\mathrm{C}$ or the buffer (Figure 1). Since a too-low MIC number was considered to carry a risk of recipient sensitization (recipients in group A received only $1 \%$ of the number of MICs compared with patients in groups B and C), MICs were administered only 2 days before transplantation during the dose escalation phase. From group $\mathrm{B}$ to group $\mathrm{C}$, the administration time point was changed from day -2 to day -7 before transplantation. Based on preclinical experiments, day -7 was considered the optimal time of administration.

Donor visit number 3 (end of study) was on the day after leukapheresis (day-1 in D1-D6 and day-6 in D7, D11, D12, and D14). During recipient visit number 3 on day -1 before transplantation surgery, recipient sensitization by the MIC product was excluded by CDC and ELISA crossmatching and HLA Ab screening using the Luminex technique (One Lambda). Transplant recipient post-transplantation visits took place on day $7 \pm 1$ (visit 4 , including a protocol biopsy procedure) and day $30 \pm 4$ (visit 5 , end of study).

Maintenance immunosuppressive therapy and post-transplantation care were performed according to center practices, with CyA, EC-MPS, and methylprednisolone administered starting on the day of surgery. Detailed information on the immunosuppressive therapy for patients is provided in Supplemental Figure 3. Ab induction therapy with an IL-2 receptor antagonist was not performed in MIC-treated patients, since IL-2 receptor signaling is believed to be critical for the development and expansion of regulatory cells (27).

Follow-up period and transplanted controls. After the study endpoint on day 30, patients were followed out to day 360 after kidney 
transplantation, with regular outpatient visits according to the center's practices. Routine screening was performed for BKV (on every outpatient visit throughout year 1) and DSAs (days 7, 30, 180, and 360 and additional screening for TOL-1 patients on days $60,90,135$, and 270). During follow-up, biomaterials were collected and analyzed retrospectively. Immunosuppressive therapy for patients in group C was reduced to lower CyA and EC-MPS doses with or without corticosteroids during follow-up beyond day 30 to avoid infectious complications of combined cell-based and immunosuppressive therapy (Supplemental Figure 3).

Details on the transplanted controls are provided in the Supplemental Methods.

MIC product manufacturing, quality control, and potency assay. Details regarding MIC product manufacturing, quality control, and the potency assay are given in the Supplemental Methods and Supplemental Tables 10-12.

Further investigations. Immunological testing, quantitative chimerism analysis in peripheral blood, proteomics analysis of patients' serum samples, and gene expression analysis of peripheral blood were performed as described in detail in the Supplemental Methods.

Statistics. Because of the small number of patients and the exploratory approach of the trial, only descriptive statistical methods were applied. In the text and tables, continuous data are summarized as the median and the range and categorical data as absolute and relative frequencies. Longitudinal data are displayed over time, stratified by group. In figures, the results are presented as individual measurements (and the median) or the median and interquartile range. The methods of analysis of proteome and gene expression data are described in the Supplemental Methods.

Study approval. The TOL-1 study was reviewed and approved by the ethics committee of the University of Heidelberg, Heidelberg, Germany, and the Paul-Ehrlich Institute, Langen, Germany (ethics number: AFmo-549/2014; Paul-Ehrlich Institute, Vorlagen-Nr. 2252/01; EudraCT number: 2014-002086-30; Clinicaltrials.gov identifier: NCT02560220). The study was performed in compliance with the provisions of the Declaration of Helsinki and Good Clinical Practice guidelines. Written informed consent was obtained from participants before inclusion in the study. Biomaterials were collected and analyzed according to protocols approved by the ethics committee of the University of Heidelberg (ethics numbers: 082/2005, 083/2005, S-395/2011, and S-225/2014).

\section{Author contributions}

CM, A. Schmitt, CK, GO, C. Sommerer, DC, MZ, M. Schmitt, M. Schaier, and PT designed the study. CM, MZ, and M. Schaier recruited participants. A. Schmitt, CK, LW, MN, AHK, and M. Schmitt produced MICsunder good manufacturing practice (GMP) conditions and were responsible for quality control of the prod- ucts. CM, FK, C. Speer, CN, LPDS, C. Sommerer, and M. Schaier collected the data. CM, A. Schmitt, CK, VD, C. Süsal, EI, LW, MN, AHK, UM, AM, CE, RW, PS, CMT, JDH, SAM, MSSA, ASB, JR, M. Schmitt, M. Schaier, and PT analyzed the data. A. Sander and $\mathrm{MH}$ advised on the statistical analysis and reviewed the manuscript. CM, A. Schmitt, VD, GO, C. Süsal, MZ, M. Schmitt, M. Schaier, and PT interpreted the data. CM and PT designed the figures. CM, A. Schmitt, GO, and PT wrote the first draft of the manuscript, and all authors revised it critically and approved the final version. Cofirst authorship of CM and A. Schmitt and co-senior authorship of M. Schaier and PT, as well as the respective order of their names, had already been defined in the clinical study protocol. The order of the co-first authors' names was determined on the basis of their contributions to the study.

\section{Acknowledgments}

We are grateful to all the patients and donors who were initially screened and recruited, especially those who actually participated in the clinical trial and without whom the TOL-1 study would not have been possible. We thank Birgit Michels, Ulrike Gern, Mandy Hinkelbein, Stefanie Mechler, Petra Richter, Brigitte Neuber, Alexander Kunz (GMP Core Facility), Volker Eckstein (FACS Core Facility), Renate Schulz, and Sandra Kräker (Chimerism Laboratory) of the Department of Hematology; Marita Heilke, Silja Petersen-Nowag, Marion Miltz-Savidis, Martina Kutsche-Bauer, Regina Seemuth, Anja Brüchig, Tina Hildenbrand, Marzena Kirschke, Olga Ochs, and Sibylle Bassauer (Transplantation Immunology) of the Institute of Immunology; Gabriela Lange, Ute von der Emde, Iris Arnold, Anke Wollschläger, and Sabine Böhnisch-Schmitt of the Department of Nephrology (all at Heidelberg University Hospital, Heidelberg, Germany); and Petra Pavel at the Institute for Clinical Transfusion Medicine and Cell Therapy (Heidelberg, Germany). We thank Peter Schnülle, (Nierenzentrum Weinheim, Weinheim, Germany) and Frieder Keller (Division of Nephrology, University Hospital Ulm, Ulm, Germany) for data safety monitoring. We would also like to thank Amy Publicover of the Department of Hematology, Southampton University Hospitals Trust (University of Southampton, Southampton, United Kingdom) for reviewing the manuscript. This study was funded by EXIST Transfer of Research I (03EFBBW056) and II (O3EUBBW056) grants from the Federal Ministry for Economic Affairs and Energy (formerly the Federal Ministry for Economic Affairs and Technology) in Berlin, Germany, and by TolerogenixX GmbH in Heidelberg, Germany.

Address correspondence to: Christian Morath, Department of Nephrology, Heidelberg University Hospital, Im Neuenheimer Feld 162, 69120 Heidelberg, Germany. Phone: 49.6221.9112.207; Email: christian.morath@med.uni-heidelberg.de.
1. Sellarés J, et al. Understanding the causes of kidney transplant failure: the dominant role of antibody-mediated rejection and nonadherence. Am J Transplant. 2012;12(2):388-399.

2. Opelz G, Döhler B. Association between steroid dosage and death with a functioning graft after kidney transplantation. Am J Transplant. 2013;13(8):2096-2105.
3. Morath C, et al. Cell therapeutic approaches to immunosuppression after clinical kidney transplantation. Pediatr Nephrol. 2018;33(2):199-213.

4. Kawai T, et al. HLA-mismatched renal transplantation without maintenance immunosuppression. N Engl J Med. 2008;358(4):353-361.

5. Scandling JD, et al. Tolerance and chimerism after renal and hematopoietic-cell transplanta- tion. N Engl J Med. 2008;358(4):362-368.

6. Leventhal JR, et al. Immune reconstitution/ immunocompetence in recipients of kidney plus hematopoietic stem/facilitating cell transplants. Transplantation. 2015;99(2):288-298.

7. Eder M, et al. Allograft and patient survival after sequential HSCT and kidney transplantation from the same donor-A multicenter analysis. Am 


\section{CLINICAL MEDICINE}

J Transplant. 2019;19(2):475-487.

8. Leventhal JR, Mathew JM. Outstanding questions in transplantation: Tolerance. Am J Transplant. 2020;20(2):348-354.

9. Tang Q, Vincenti F. Transplant trials with Tregs: perils and promises. JClin Invest. 2017;127(7):2505-2512.

10. Kleist C, et al. Generation of suppressive blood cells for control of allograft rejection. Clin Sci. 2015;128(9):593-607.

11. Radu CA, et al. Mitomycin-C-treated peripheral blood mononuclear cells (PBMCs) prolong allograft survival in composite tissue allotransplantation. J Surg Res. 2012;176(2):e95-e101.

12. Dittmar L, et al. Immunosuppressive properties of mitomycin C-incubated human myeloid blood cells (MIC) in vitro. Hum Immunol. 2015;76(7):480-487.

13. Newell KA, et al. Identification of a B cell signature associated with renal transplant tolerance in humans. JClin Invest. 2010;120(6):1836-1847.

14. Silva HM, et al. Preserving the B-cell compartment favors operational tolerance in human renal transplantation. Mol Med. 2012;18:733-743.

15. Chesneau M, et al. Unique B cell differentiation profile in tolerant kidney transplant patients. Am J Transplant. 2014;14(1):144-155.
16. Shabir S, et al. Transitional B lymphocytes are associated with protection from kidney allograft rejection: a prospective study. Am J Transplant. 2015;15(5):1384-1391.

17. Tebbe B, et al. Renal transplant recipients treated with calcineurin-inhibitors lack circulating immature transitional $\mathrm{CD} 19^{+} \mathrm{CD} 24^{\mathrm{hi}}$ CD $38^{\text {hi }}$ regulatory B-lymphocytes. PLOS ONE. 2016;11(4):e0153170.

18. Svachova V, et al. Dynamic changes of B-cell compartments in kidney transplantation: lack of transitional B cells is associated with allograft rejection. Transpl Int. 2016;29(5):540-548.

19. Rosser EC, Mauri C. Regulatory B cells: origin, phenotype, and function. Immunity. 2015;42(4):607-612.

20. Nova-Lamperti E, et al. IL-10-produced by human transitional B-cells down-regulates CD86 expression on B-cells leading to inhibition of CD4+T-cell responses. Sci Rep. 2016;6:20044.

21. Bottomley MJ, Chen M, Fuggle S, Harden PN, Wood KJ. Application of operational tolerance signatures are limited by variability and type of immunosuppression in renal transplant recipients: a cross-sectional study. Transplant Direct. 2017;3(1):e125.

22. Rebollo-Mesa I, et al. Biomarkers of tolerance in kidney transplantation: are we predicting tolerance or response to immunosuppressive treatment? Am J Transplant. 2016;16(12):3443-3457.

23. Spitzer TR, et al. Twenty-year follow-up of histocompatibility leukocyte antigen-matched kidney and bone marrow cotransplantation for multiple myeloma with end-stage renal disease: lessons learned. Transplantation. 2019;103(11):2366-2372.

24. Leventhal JR, Ildstad ST. Tolerance induction in HLA disparate living donor kidney transplantation by facilitating cell-enriched donor stem cell Infusion: The importance of durable chimerism. Hum Immunol. 2018;79(5):272-276.

25. Scandling JD, et al. Chimerism, graft survival, and withdrawal of immunosuppressive drugs in HLA matched and mismatched patients after living donor kidney and hematopoietic cell transplantation. Am J Transplant. 2015;15(3):695-704.

26. Radu CA, et al. The combination of mitomycininduced blood cells with a temporary treatment of ciclosporin A prolongs allograft survival in vascularized composite allotransplantation. Langenbecks Arch Surg. 2018;403(1):83-92.

27. Nelson BH. IL-2, regulatory T cells, and tolerance. JImmunol. 2004;172(7):3983-3988. 